
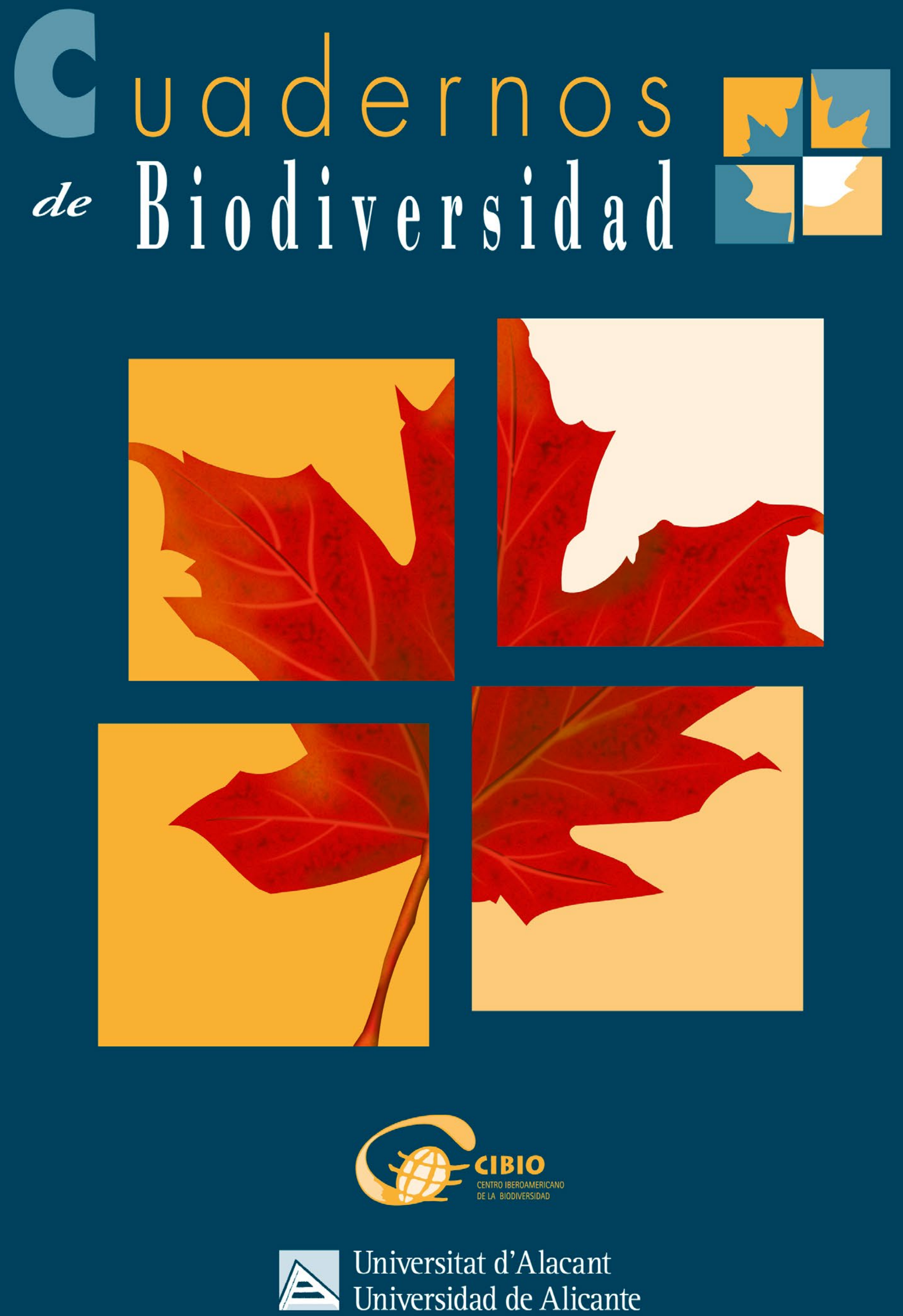


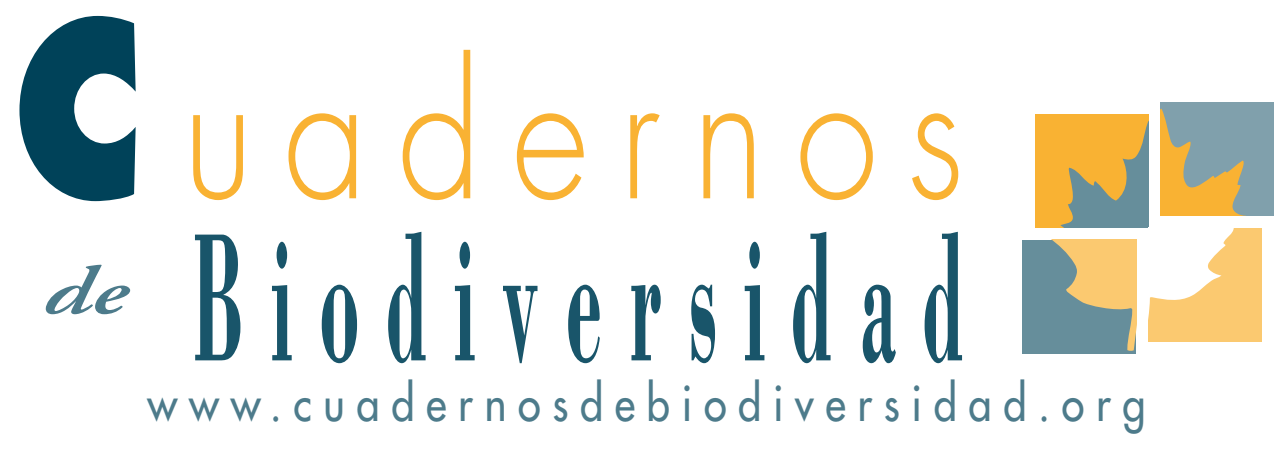

\section{Entomofauna asociada a las plantas del campus de la Universidad de Alicante (España)}

\section{Entomofauna associated the plants of the campus of the University of Alicante (Spain)}

\section{K. Hernández-Martínez, M.A Marcos García}

Instituto de Investigación CIBIO, Universidad de Alicante. Crta. San Vicente del Raspeig s/n Alicante, España 03003

kevinhdezmrtz@gmail.com marcos@ua.es

\section{RESUMEN}

La entomofauna de los espacios verdes urbanos es poco conocida aun siendo reservorio de insectos plaga y beneficiosos. Este estudio tiene como principal objetivo catalogar los insectos plaga y sus potenciales enemigos naturales con el propósito de facilitar información para un correcto manejo integrado de este ecosistema ajardinado. Se realizaron muestreos durante 6 meses que dieron como resultado el registro de interacciones entre niveles tróficos así como la identificación de 69 especies, 53 insectos fitófagos potencialmente plaga o polinizadores pertenecientes a 7 órdenes y 28 familias (áfidos, trips, coleópteros, cochinillas, psílidos, aleuródidos, àpidos, minadoras de hojas y frutos... ), 2 especies de ácaros potencialemente plaga así como 14 enemigos naturales, depredadores o parasitoides, pertenecientes a 5 órdenes y 8 familias. Podemos concluir que la entomofauna fitófaga se encuentra bien controlada, en general, por sus enemigos naturales y que participan en una amplia red de interacciones tritróficas: planta-fitófago-enemigo natural cuyo conocimiento es crucial para el establecimiento de estrategias acertadas de control integrado de plagas en estos ecosistemas urbanos donde el uso de productos fitosanitarios está cada día mas restringido. 
Pallabras clave: fitófagos, plaga, polinizadores, espacios verdes urbanos, interacciones plantainsecto, control biológico.

\section{ABSTRACT}

The entomofauna of the urban greenspaces is little known even being a reservoir of plague and beneficial insects. The main objective of this study is to catalogue pest insects and their potential natural enemies with the purpose of providing information for a correct integrated management of this landscaped ecosystem. Samples were carried out during 6 months that resulted in the recording of interactions between trophic levels as well as the identification of 69 species, 53 phytophagous insects potentially plague or pollinators belonging to 7 orders and 28 families (aphids, thrips, coleoptera, cochineals, psyllids, aleurodids, bees, leaf and fruit miners...), 2 species of potentially plague mites as well as 14 natural enemies, predators or parasitoids, belonging to 5 orders and 8 families. We can conclude that the entomofauna phytophaga is well controlled, in general, by its natural enemies and that they participate in a wide network of tritrophic interactions: plantphytophagous-natural enemy whose knowledge is crucial for the establishment of successful strategies of integrated pest control in these urban ecosystems where the use of phytosanitary products is increasingly restricted.

Key words: phytophagous, pest, pollinator, urban greenspaces, insect-plant interacctions, biological control.

\section{INTRODUCCIÓN}

Los espacios verdes urbanos son pequeños ecosistemas, aunque fragmentados, suponen un reservorio de biodiversidad (Egerer et al., 2018) donde encontramos especies vegetales ornamentales, seleccionadas con un fín estético, así animales, sobretodo entomofauna. Para su buena gestión y manejo es imprescindible conocer las especies que lo componen y sus interacciones por lo que se hace muestreos. A diferencia de los cultivos agrícolas donde se realizan muestreos sistemáticos, normalmente de un monocultivo, en los espacios verdes urbanos se requieren otros más especializados donde gran parte de la eficacia depende de la experiencia del técnico observador.

Los muestreos tienen como fin identificar las especies fitófagas que debido a su alto potencial reproductivo toman carácter de plaga provocan graves daños en las plantas de las que se alimentan, pérdidas económicas por deterioro del moviliario urbano, molestias a los usuarios, y en casos muy puntuales, pueden afectar de manera negativa a la salud de las personas o de animales domésticos (García et al., 2015) así como las especies beneficiosas que actúan como enemigos naturales de estas.

Con este estudio se pretende caracterizar la entomofauna fitófaga (nivel trófico 2) de las zonas ajardinadas del campus de la Universidad de Alicante, así como establecer las interacciones con sus plantas huésped (nivel trófico 1) y con sus enemigos naturales (nivel trófico 3) con el objetivo de servir como referencia para la mejora de las estrategias de gestión y manejo estas zonas ajardinadas, así como para el control biológico e integrado de sus plagas, entendiendo este último como "la estrategia para combatir las plagas y enfermedades mediante el uso de todos los métodos disponibles, a la vez que se minimiza la aplicación de plaguicidas de origen químico (Stenberg, 2017)”.

\section{MATERIAL Y MÉTODOS}

El estudio se realizó en el área ajardinada del campus de la Universidad de Alicante ubicado en el término de San Vicente del Raspeig, Alicante, SE Península Ibérica $\left(38^{\circ} 23^{\prime} 15^{\prime \prime} \mathrm{N} 0^{\circ} 30^{\prime} 53^{\prime \prime} \mathrm{W}\right)$ a una altitud de $92 \mathrm{~m}$. Se estudiaron especies arbóreas y arbustivas tipicamente forestales, árboles frutales propios de climas mediterráneos (cítricos, olivos, granado, etc), plantas ornamentales propias de ecosistemas áridos y tropicales, así como diferentes especies de un huerto urbano con diferentes especies hortícolas. 
Durante 6 meses (noviembre 2017-mayo 2018), se realizaron muestreos mensuales prospectando las especies vegetales de porte arbóreo y arbustivo así como las hortícolas, teniendo en cuenta las singularidades de las diferentes zonas de vegetación. La información botánica y ubicación de algunas de estas especies se obtuvo a partir del "Inventario de las especies vegetales del campus" realizado por la empresa encargada de la gestión y manejo de la zona, las restantes se localizaron.

En campo las muestras prospectadas eran introducidas en pequeños recipientes con alcohol al $70 \%$ o bien guardadas en bolsas de plástico individualizadas para cada registro y acompañadas de una etiqueta con la información pertinente (ubicación dentro del campus, fecha y planta huésped), donde fueron preservadas hasta su identificación. Algunas muestras se mantuvieron vivas en cajas de cría hasta su desarrollo como adulto. Las muestras se encuentran depositadas en la colección Entomológica del CIBIO en la Universidad de Alicante (CEUA).

Para la identificación de las muestras se utilizó un microscopio estereoscópico con iluminación incorporada (Leica MZ95) y bibliografía especializada en cada grupo (Climent, 1990a; Climent, 1990b; Climent, 1992; García-Marí et al., 1991; De Liñan et al., 2001: Bellmann, 2003: Barrientos et al., 2004: Bignon, 2006: Moret y Martí, 2006: Leraut, 2007: Muñoz López et al., 2011). También se utilizaron páginas web (Bermejo, 2011; Chueca y Moltó, 2012; Parr et al., 2014; CABI, 2018; Myers et al., 2018). Algunas muestras fueron revisadas por entomólogos especialistas (ver agradecimientos) a partir de fotografías hechas tanto en campo en el momento de la recogida de muestras o del análisis de las muestras en el laboratorio para verificar la identificación. Las especies identificadas se clasificaron en fitófagas y enemigos naturales. Por fitófagas entendemos aquellas especies que se alimentan de algún tejido vegetal vivo, por enemigos naturales entendemos aquellos insectos depredadores o parasitoides de las especies fitófagas y que por lo tanto pueden controlar las poblaciones de las especies plaga, independientemente de que alguna de sus fases de desarrollo pueda alimentarse de material vegetal. Depredadores entendemos como aquellas especies de vida libre en las que alguna de sus fases de desarrollo es insectívora y caza varias presas durante su desarrollo y parasitoides aquellas especies cuyos adultos son de vida libre, pero sus fases inmaduras se desarrollan a expensas de un único insecto huésped provocándole la muerte.

Los mapas de interacciones planta-insecto y fitófago-enemigo natural se realizaron con R-project usando el paquete bipartite.

\section{RESULTADOS}

Se identificaron un total de 69 especies diferentes de artrópodos. 55 de ellas clasificadas como fitófagas al destacar primordialmente por alimentarse de y 14 especies clasificadas como enemigos naturales por depredar o parasitar en alguna de sus estadios alguna de las especies anteriores.

\section{Especies fitófagas}

Las especies fitófagas pertenecen a 7 órdenes y 28 familias de insectos, y a 2 especies de quelicerados representados por ácaros de la familia Tetranychidae. En la tabla 1 se presenta su clasificación taxonómicamente y en la figura 1 se registran las interacciones con las plantas huéspedes observadas en el campus. 
Tabla 1. Especies fitófagas registradas y sus plantas huésped en la zona ajardinada del campus de la Universidad de Alicante. $\mathrm{Ga}=$ gallícola $\mathrm{Po}=$ Polinizador

\begin{tabular}{|c|c|c|}
\hline Orden & Familia & Especie o Género \\
\hline \multirow[t]{5}{*}{ Coleoptera } & Chrysomelidae & Xanthogaleruca luteola (Müller, 1776) \\
\hline & \multirow[t]{2}{*}{ Curculionidae } & Otiorhynchus cribricollis (Gyllenhal, 1834) \\
\hline & & Rhynchophorus ferrugineus (Olivier, 1790) \\
\hline & Scolytidae & Tomicus sp \\
\hline & Scarabaeidae & Oxythyrea funesta (Poda, 1761) \\
\hline \multirow[t]{3}{*}{ Diptera } & Agromyzidae & Liriomyza sp \\
\hline & \multirow{2}{*}{ Tephritidae } & Bactrocera oleae (Rossi, 1790) \\
\hline & & Ceratitis capitata (Wiedemann, 1824) \\
\hline \multirow[t]{22}{*}{ Hemiptera } & \multirow[t]{2}{*}{ Aleyrodidae } & Aleurothrixus floccosus (Maskell,1896) \\
\hline & & Paraleyrodes minei Laccarino, 1990 \\
\hline & \multirow[t]{10}{*}{ Aphididae } & Aphis fabae Scopoli, 1763 \\
\hline & & Aphis gossypii Glover, 1877 \\
\hline & & Aphis nerii Boyer de Fonscolombe, 1841 \\
\hline & & Aphis punicae Passerini, 1863 \\
\hline & & Brevicoryne brassicae (Linneaus, 1758) \\
\hline & & Capitophorus elaeagni (Del Guercio, 1894) \\
\hline & & Greenidea ficicola Takahashi,1921 \\
\hline & & Hoplocallis pictus (Ferrari, 1872) \\
\hline & & Myzus persicae (Sulzer, 1776) \\
\hline & & Nasonovia ribisnigri (Mosley,1841) \\
\hline & Cicadellidae & Empoasca sp \\
\hline & \multirow[t]{4}{*}{ Coccidae } & Ceroplastes rusci (Linnaeus, 1758) \\
\hline & & Icerya purchasi (Maskell, 1878) \\
\hline & & Protopulvinaria pyriformis (Cockerell, 1894) \\
\hline & & Saissetia oleae (Olivier, 1791) \\
\hline & Dactylopiidae & Dactylopius opuntiae (Cockerell) \\
\hline & \multirow[t]{3}{*}{ Diaspididae } & Aonidiella aurantii (Maskell,1896) \\
\hline & & Carulaspis juniperi (Bouché, 1833) \\
\hline & & Lepidosaphes beckii (Packard,1869) \\
\hline & Kermesidae & Kermes vermilio (Planchon, 1864) \\
\hline
\end{tabular}




\begin{tabular}{|c|c|c|}
\hline & & \\
\hline & Lygaeidae & Oxycarenus lavaterae (Fabricius, 1787) \\
\hline & Pemphigidae & Forda riccobonii De Stefani, 1899 \\
\hline & Pentatomidae & Nezara viridula (Linneaus, 1758) \\
\hline & \multirow[t]{3}{*}{ Pseudococcidae } & Planococcus citri Risso, 1813 \\
\hline & & Pseudococcus longispinus (Targioni Tozzetti, 1867) \\
\hline & & Pseudococcus sp \\
\hline & \multirow[t]{4}{*}{ Psyllidae } & Cacopsylla pulchella (Löw,1877) \\
\hline & & Euphyllura olivina Costa 1839 \\
\hline & & Glycaspis brimblecombei Moore, 1964 \\
\hline & & Platycorypha nigrivirga Burckhardt, 1987 \\
\hline & Psylloidae & Macrohomotoma gladiata Kuwayama, 1908 \\
\hline \multirow[t]{8}{*}{ Hymenoptera } & \multirow[t]{2}{*}{ Agaonidae $(\mathrm{Ga})$} & Blastophaga psenes (Linneaus, 1758) \\
\hline & & Josephiella microcarpae Beardsley \& Rasplus, 2001 \\
\hline & Andrenidae (Po) & Andrena sp \\
\hline & \multirow[t]{2}{*}{ Apidae (Po) } & Nomada sp \\
\hline & & Xylocopa violacea (Linnaeus, 1758) \\
\hline & Cynipidae $(\mathrm{Ga})$ & Plagiotrochus australis (Mayr, 1882) \\
\hline & \multirow[t]{2}{*}{ Eulophidae (Ga) } & Leptocybe invasa Fisher \& La Salle, 2004 \\
\hline & & Ophelimus maskelli (Ashmead, 1900) \\
\hline \multirow[t]{2}{*}{ Lepidoptera } & Gracillariidae & Phyllocnistis citrella Stainton, 1856 \\
\hline & Licenidae & Cacyreus marshalli Butler, 1897 \\
\hline Orthoptera & Acrididae & Anacridium aegyptium Linnaeus, 1758 \\
\hline Thysanoptera & Phlaeothripidae & Gynaikothrips ficorum (Marchal, 1908) \\
\hline \multirow[t]{2}{*}{ Trombidiformes } & \multirow[t]{2}{*}{ Tetranychidae } & Panonychus citri McGregor, 1878 \\
\hline & & Tetranychus urticae Koch,1836 \\
\hline
\end{tabular}


Figura 1. Mapa de interacciones entre la planta huesped y el insecto fitófago

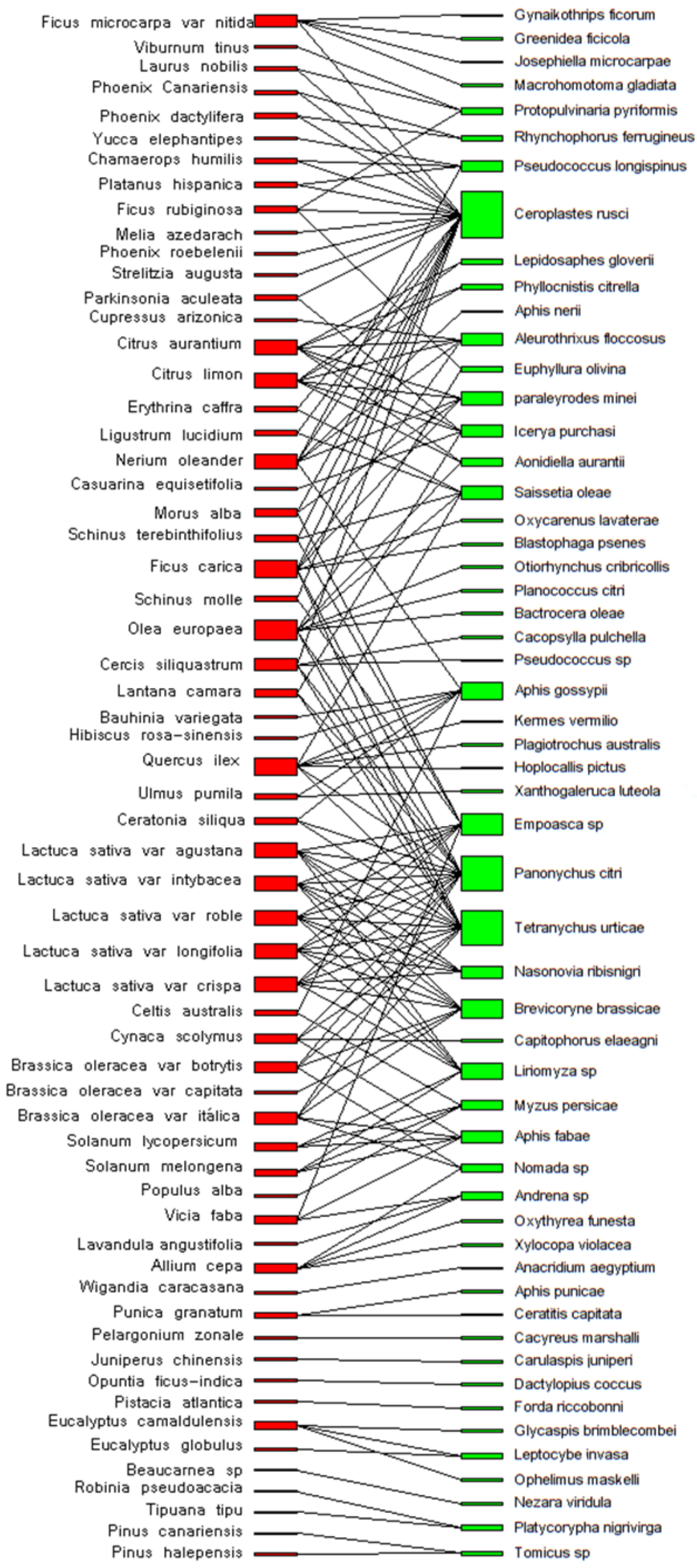




\section{Enemigos naturales}

Respecto a las especies de enemigos naturales que constituyen el tercer nivel trófico, se registraron representantes de 5 órdenes y 8 familias de insectos (Tabla 2) y las interacciones con los fitófagos a los que depredaran o parasitan asi como si alguno de sus estadios se considera polinizador (Figura 2).

Tabla 2. Enemigos naturales y polinizadores identificados en la zona ajardinada del campus. $\mathrm{D}=$ Depredador, $\mathrm{Pa}=$ Parasitoide, $\mathrm{Po}=$ Polinizador

\begin{tabular}{|c|c|c|}
\hline Orden & Familia & Especie fitógaga \\
\hline Coleoptera & Coccinellidae (D) & Adalia decempunctata (Linneaus 1758) \\
\hline \multirow[t]{6}{*}{ Diptera } & \multirow[t]{6}{*}{ Syrphidae (D, Po) } & Eupeodes corollae (Fabricius, 1794) \\
\hline & & Episyrphus balteatus (De Geer, 1776) \\
\hline & & Paragus haemorrhous Meigen, 1822 \\
\hline & & Scaeva albormaculata (Macquart, 1842) \\
\hline & & Sphaerophoria rueppellii (Wiedemann, 1820) \\
\hline & & Sphaerophoria scripta (Linnaeus, 1758) \\
\hline Hemiptera & Miridae (D) & Nesidiocoris tenuis (Reuter, 1895) \\
\hline \multirow[t]{5}{*}{ Hymenoptera } & Aphididae $(\mathrm{Pa})$ & Aphidius sp \\
\hline & \multirow[t]{2}{*}{ Braconidae $(\mathrm{Pa})$} & Diaeretiella rapae (M'Intosh, 1855) \\
\hline & & Trioxys curvicaudus Mackauer, 1967 \\
\hline & Encyrtidae $(\mathrm{Pa})$ & Methaphycus helvolus (Compere, 1926) \\
\hline & Torymidae $(\mathrm{Pa})$ & Torymus notatus (Walker, 1833) \\
\hline Neuroptera & Chrysopidae (D) & Chrysoperla carnea (Stephens, 1836) \\
\hline
\end{tabular}


Figura 2. Mapa de interacciones entre los fitófagos y los enemigos naturales

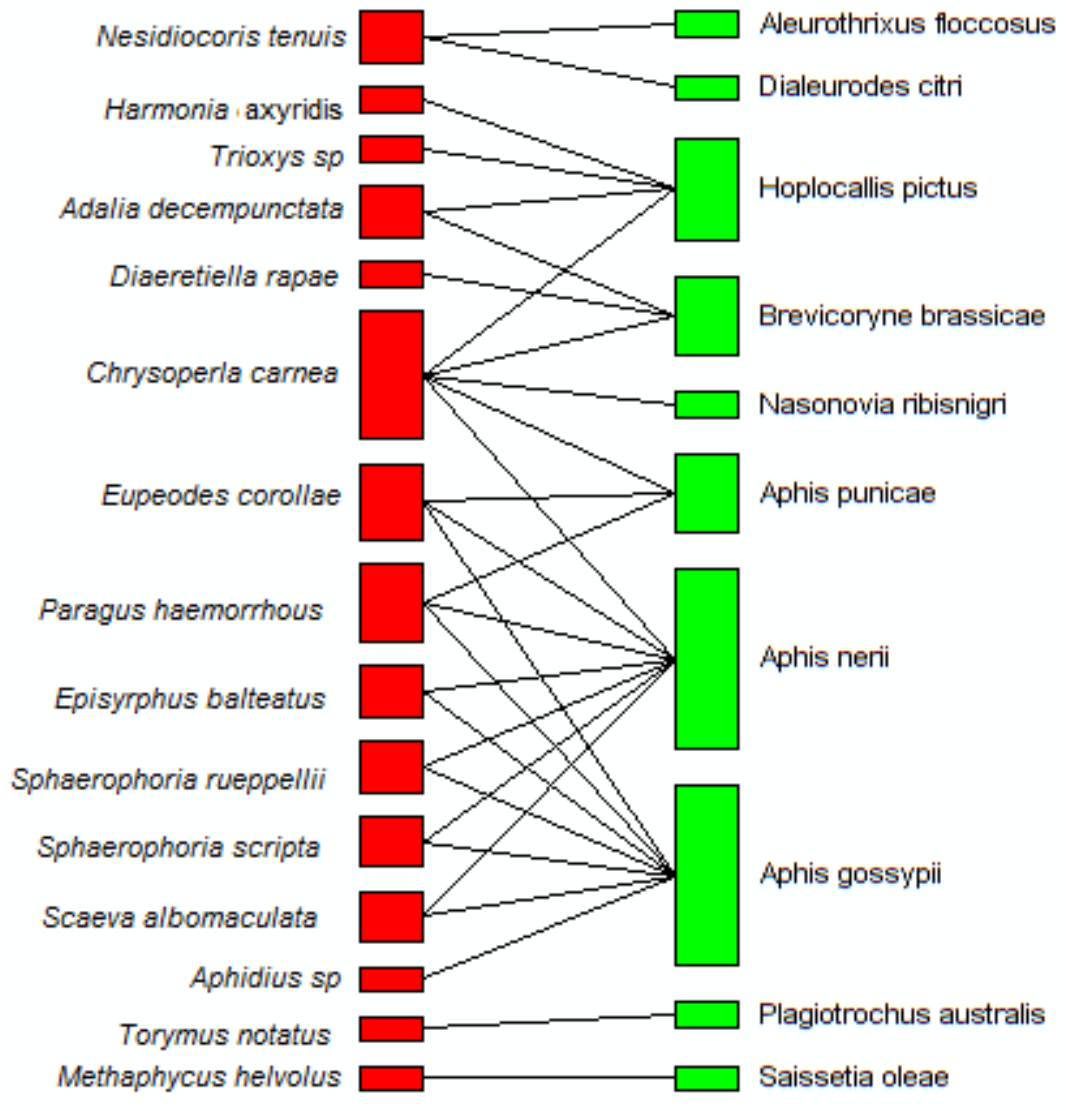

Entre estos enemigos naturales destaca la presencia en el campus de 4 variaciones del patrón de color de los élitros de la especie Adalia decempunctata (Figura 3) y de las agallas en forma de media luna de Forda ricobonii en Pistacia atlantica (Figura 4)

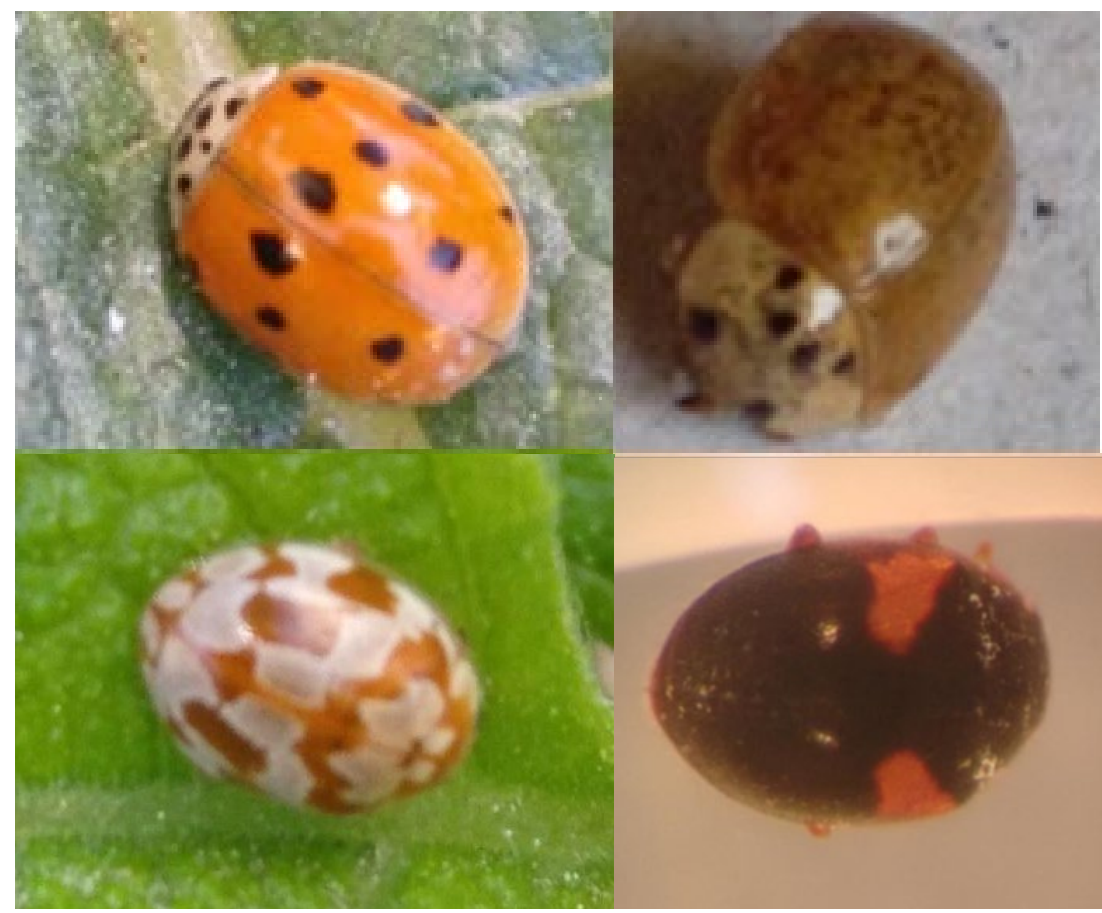

Figura 3. Variaciones del patrón de color de los élitros de Adalia decempunctata encontrados. 


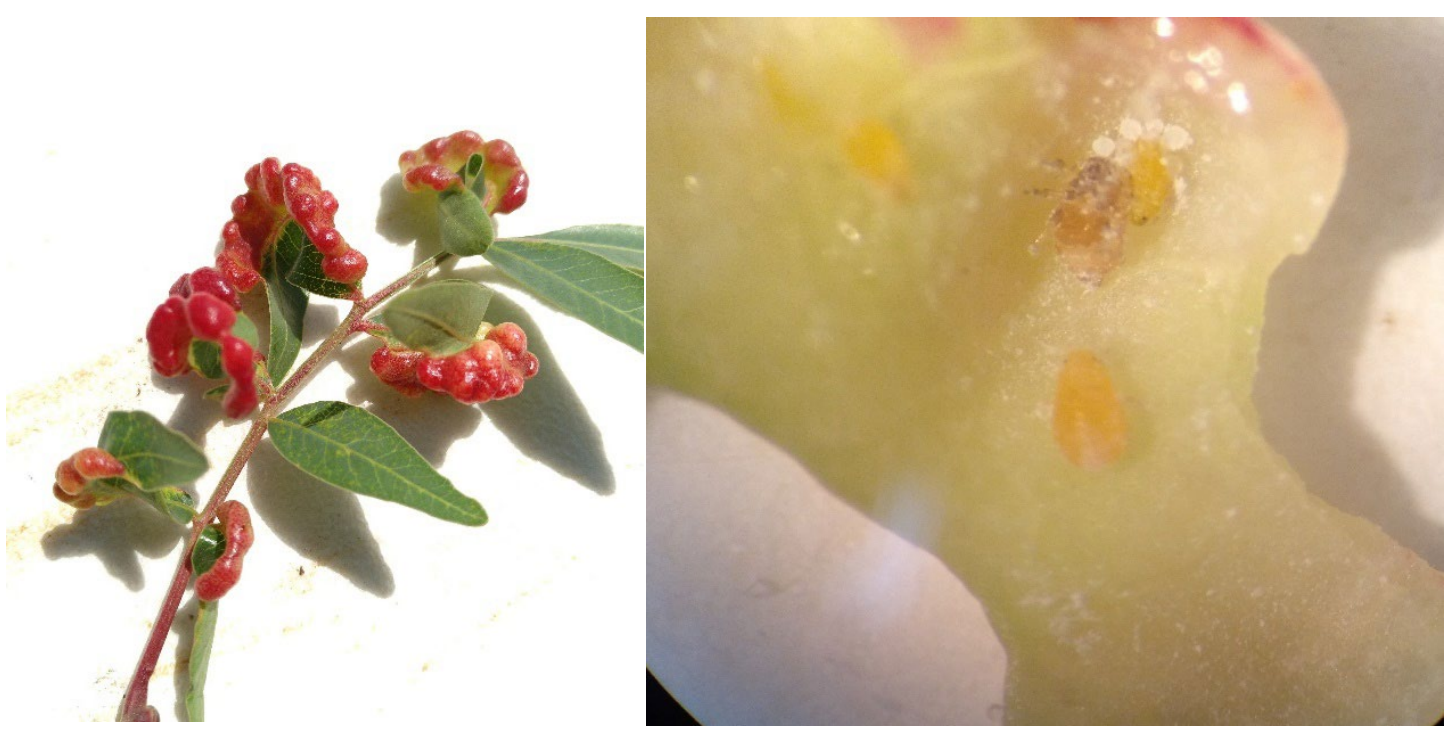

Figura 4. Agallas formadas por Forda riccobonii en Pistacia atlantica e interior de estas.

\section{DISCUSIÓN}

La gran diversidad de insectos encontrados en las zonas verdes del campus de la Universidad de Alicante indica su importancia como reservorio de biodiversidad, tanto de especies potencialmente plaga, como de sus enemigos naturales y polinizadores. También se han registrado dos especies de ácaros, Panonychus citri y Tetranychus urticae, en diversas especies vegetales que ocasionan daños y pueden llegar a ser plagas de árboles frutales, principalmente de cítricos y de hortalizas (Garcia-Marí et al., 1991)

Entre las interacciones insecto-planta registradas (Figura 1), podemos distinguir aquellas que presentan una alta especificidad respecto al huésped como por ejemplo el trips Gynaikothrips ficorum que sólo se ha registrado alimentándose de las células foliares de Ficus microcarpa var nitida L.f. pese a haber otras especies huésped del campus de este género como $F$. benjamina L., F. elastica Roxb. ex Hornem., u otras especies arbóreas del género Citrus que parecen no estar afectadas, por el momento, por este tisanóptero (Denmark et al., 2004). El agaónido Josephiella microcarpae también es específico de Ficus microcarpa produciendo unas agallas características en las hojas. Esta especie fue registrada por vez primera en
España en 2001 en las islas Canarias, pero no fue hasta 2017 cuando se registró la primera población en el área peninsular (Rodrigo et al., 2017; Rodrigo Santamalia et al., 2011) y a partir de ahí se ha ido extendiendo por toda la península. El coleóptero Xanthogaleruca luteola lo encontramos asociado a Ulmus pumila L. pudiendo también estar asociado a otras especies de olmos europeos. Las especies asiáticas y americanas de Ulmus en ensayos de laboratorio han demostrado ser también susceptibles a este crisomélido como huésped potencial, siendo Ulmus wilsoniana L. la única opción para evitar el ataque en espacios verdes urbanos (Hall y Young, 1986; Hall y Townsend, 1987). De las dos especies de cochinillas encontradas, Kermes vermilio (antiguamente utilizada para la extracción de colorante natural) se alimenta sólo sobre encina (Quercus ilex L.) y coscoja (Quercus coccifera L.) (Pellizzari et al., 2012) al igual que Dactylopius opuntiae asociada exclusivamente a Opuntia ficus-indica (L) Mill. aunque con registros sobre otras especies del género Opuntia (ChávezMoreno et al., 2011). En cuanto a las especies de psílidos también se registraron interacciones específicas alimentándose siempre de fluidos vegetales de las siguientes especies arbóreas: Cacopsylla pulchella sobre Cercis siliquastrum L., Macrohomotoma gladiata sobre hojas de Ficus microcarpa var nitida, 
Euphyllura olivina sobre parte apical de ramas de olivo (Olea europaea L.), Platycorypha nigrivirga sobre Tipuana tipu (Benth.) O. Kuntze, y Glycaspis brimblecombe sobre Eucalyptus camaldulensis Dehn. En Eucalyptus camaldulensis también registramos relaciones específicas en las especies de avispillas gallícolas Ophelimus maskelli y Leptocybe invasa, esta última también se registraron en Eucalyptus globulus Labill., coincidiendo con las observaciones de otros investigadores (Valente y Hodkinson, 2009; Dhahri et al., 2010; Caleca, 2010).

Los áfidos son el grupo más abundante y de los más temidos por las graves pérdidas económicas que provocan como consecuencia de sus dańos directos (succión de savia y cubrir de melaza las hojas interrumpiendo parcialmente la función fotosintética) e indirectos (transmisión de virus) en mutitud de especies vegetales. Brevicoryne brassicae, conocido como el pulgón de las crucíferas, lo encontramos asociado a las diversas variedades de Brassica oleracea L., pero tiene muchos otros huéspedes potenciales (Cole, 1997). Nasonovia ribisnigri registrado en la lechuga (Lactuca sativa L.) de quien es considerada su principal plaga (Diaz y Fereres, 2005). Aphis gossypii y Myzus persicae son especies polífagas que se encuentran sobre diferentes especies de plantas ornamentales del campus. Por el contrario, Aphis nerii se ha registrado exclusivamente en la adelfa (Nerium oleander L.) en el campus al no haber otras plantas ornamentales de las familias Apocynaceae y Asclepiadaceae (Blackman y Eastop, 2000). Del mismo modo, el pulgón Capitophorus elaeagni se ha encontrado sólo sobre plantas de alcachofa (Cynara scolymus L.) aunque puede tener otros huéspedes alternativos (Blackman y Eastop, 2000). El áfido Greenidea ficicola se ha registardo en ejemplares de Ficus microcarpa var nitida y Ficus carica L., pero no en las otras especies de Ficus pese a poder ser también huéspedes potenciales (Bella et al., 2009; Kamel, 2012). Destacar que los ejemplares de esta especie encontrados en el campus de la UA constituyeron parte del material que se consideró como primera cita de esta especie en la península ibérica (Pérez Hidalgo et al., 2009). Por último, destacar tres relaciones monoespecíficas entre pulgón y planta como son el caso de Hoplocallis pictus alimentándose de savia de Quercus ilex al no haber otras especies arbóreas de este género (Aguiar, 2013), Aphis punicae sobre granado (Punica granatum L.), su principal planta huésped (Biradar, 2004), y Forda riccobonii, áfido gallícola de la familia Pemphigidae, sobre Pistacia atlantica Desf. donde produce cada año agallas de dos tipos en los foliolos de sus hojas (Pérez Hidalgo, 2015).

Las especies de cochinillas encontradas son polífagas destacando Ceroplastes rusci citada en 20 plantas diferentes y según la bibliografía a gran variedad de otras (Khasawinah y Talho, 2009) al igual que las especies de Pseudococcus que además pueden ser portadoras de virus (Douglas y Krüger, 2008). Respecto a Aonidiella aurantii, a pesar de ser escasa en el campus, se ha registrado en diversos huéspedes pudiendo llegar a ser una plaga en algunas especies de cítricos (Debach, 1959). Saissetia oleae, fue encontrada en olivo, encina y limoneros ornamentales. La cochinilla algodonosa, Planococcus citri, se ha encontrado en baja densidad poblacional en el naranjo amargo (Citrus aurantium L.), en olivo, y en Platanus hispanica Münchh, pese a existir otras especies huésped en el campus (Ben-Dov, 2011). La cochinilla acanalada, Icerya purchasi, se ha localizado en las dos especies de cítricos. Lepidosaphes beckii, conocida como "serpeta gruesa" se ha registrado sobre hojas y frutos de cítricos donde produce llegar a producir grandes impactos económicos en cultivos cercanos (Rodrigo y García-Marí, 1992). Por último, la cochinilla polífaga Protopulvinaria pyriformis sólo se ha encontrado esporádicamente en Laurus nobilis L. y Ficus rubiginosa L. y en gran abundancia sobre Viburnum tinus L. extendiéndose por casi toda su superficie foliar (Wysoki, 1987).

Las dos especies de "mosca blanca" Paraleyrodes minei y Aleurothrixus floccosus, se encontraron principalmente asociadas a los cítricos sin haber otros huéspedes potenciales en el campus.

Entre los dípteros que potencialmente pueden ser plaga, encontramos a Bactrocera oleae asociada al olivo donde dańa directamente el fruto, depreciando 
la aceituna (Nardi et al., 2005). También registramos especies minadoras de hojas del género Liriomyza Mik. en algunas plantas cultivadas en el huerto, aunque no en todas las especies potenciales (Stegmaier, 1966; Zehnder y Trumble, 1984; Johnson y Hara, 1987; Kang et al., 2009). La mosca mediterránea de la fruta, Ceratitis capitata ha sido registrada solo en el fruto del granado aunque es muy posible que también se desarrolle en frutos maduros de Citrus aurantium o Ficus carica (Thomas et al., 2013).

El microlepidóptero Phyllocnistis citrella, conocido como "minador de los cítricos", lo encontramos en baja proporción minando hojas de las especies de Citrus, principalmente limoneros, aunque también mina hojas de otras especies de Rutaceas (Heppner, 1993).

La chinche verde, Nezara viridula, conocida como "pudenta" por su olor desagradable se ha encontrado sobre la palmera Beaucarnea sp. y aunque es polífaga, este no es un huésped habitual (Velasco y Walter, 1992), por lo que esta relación posiblemente no responda a una actividad trófica.

El ortóptero Anacridium aegyptium lo hemos registrado alimentándose de hojas de Wigandia caracasana Brand, aunque hay evidencias de otros años en los que también se le ha visto sobre otras plantas ornamentales que son también palatables.

Las dos especies de abejas de los géneros Andrena y Nomada, el ápido Xylocopa violacea y el coleóptero Oxythyrea funesta, se alimentan del néctar o polen de multitud de plantas por lo que podemos considerarlos como polinizadores generalistas.

También encontramos registros de interaccion procedentes de huellas o marcas de herbivoría como son los característicos orificios de su puesta en los higos de Ficus carica por parte de la avispilla Blastophaga psenes. En los olivos observamos las frecuentes señales de herbivoría en los márgenes festoneados de las hojas características del curculiónido Otiorhynchus cribricollis, pero ningún síntoma en otras plantas ornamentales y frutícolas que también son sus huéspedes potenciales (Fisher, 2007). Del barrenador del geranio Cacyreus marshalli observamos muestras de las galerias escavadas por sus larvas y los destrozos en los capullos florales sobre la especie de geranio Pelargonium zonale L. De los coleópteros barrenadores Rhynchophorus ferrugineus y especies del género Tomicus se registran sus galerías como testimonio de su daño en los troncos de varias especies de palmeras (Phoenix dactylifera L. y Phoenix canariensis Chaub.) y de pinos (Pinus halepensis Mill. y Pinus canariensis Smith), respectivamente, pero debido a los tratamientos de endoterapia que se aplican en el campus, no se han visualizado ejemplares adultos de estas especies ni de Thaumetopoea pityocampa, la procesionaria del pino, pese a haber estado presente en el campus en años anteriores ( $\mathrm{M}^{\mathrm{a}}$ Ángeles Marcos García com. pers).

Respecto a las interacciones enemigo naturalpresa encontradas (Figura 2), se ha catalogado la especie Adalia decempunctata interaccionando con los áfidos Hoplocallis pictus y Brevicoryne brassicae, aunque es depredadora generalista de otras especies de áfidos (Dixon, 1958).

De las 6 especies de sírfidos depredadores encontradas (Eupeodes corollae, Episyrphus balteatus, Episyrphus balteatus, Paragus haemorrhous, Scaeva albormaculata, Sphaerophoria rueppellii y S. scripta) se ha registrado depredación sobre al menos dos especeis de áfidos, aunque las larvas depredadoras de estas especies de sírfidos, son polífagas y capaces de alimentarse de otras muchas especies de pulgones, así como de otros artrópodos de cuerpo blando (Rojo et al., 2003). Destacar la presencia de Sphaerophoria rueppellii, especie ya comercializada para el control de plagas de áfidos.Además, los adultos de todas estas especies son polinizadores ya que visitan las flores asiduamente para alimentarse de su polen y néctar (Rotheray and Gilbert, 2011).

De la especie de hemíptero Nesidicoris tenuis se catalogó interacción con las dos especies de moscas blancas identificadas Aleurothrixus floccosus y Paraleyrodes minei, aunque tiene otras muchas presas alternativas como Tuta absoluta, Bemisia tabaci, trips, áfidos, lepidópteros, ácaros entre otras, pudiendo ser también herbívoro en ausencia de presas. El hecho 
de ser zoofitófago, hace que como enemigo natural deba tratarse con precaución ya que eventualmente puede afectar negativamente al cultivo (Sánchez, 2008; Calvo et al., 2008: Urbaneja et al., 2009)

Del Neuróptero Chrysoperla carnea registramos la interacción de sus larvas mandibuladas con 4 especies de áfidos: Hoplocallis pictus, Nasonovia ribisnigri, Aphis nerii y Brevicoryne brassicae. Según la bibliografía se puede nutrir de un mayor número de artrópodos siendo una de las especies utilizadas en estrategias de control biológico (Senior et al., 2001)
Finalmente respecto a los parasitoides (Figura 2), todos pertenecen a diversas especies de Hymenoptera: el género Aphidius, parasitoide del áfido Aphis gossypii; Methaphycus helvolus, parasitoide de la cochinilla Saissetia oleae en la que se ha podido diagnosticar por el orificio de emergencia; Torymus notatus, parasitoide de la avispilla gallícola Plagiotrochus australis y dos bracónidos; Diaeretiella rapae, parasitoide del pulgón Brevicoryne brassicae y de otros áfidos no catalogados (Pike et al., 1999), y Trioxys curvicaudus, parasitoide del áfido Hoplocallis pictus (Melia y Fernández de córdoba, 1993).
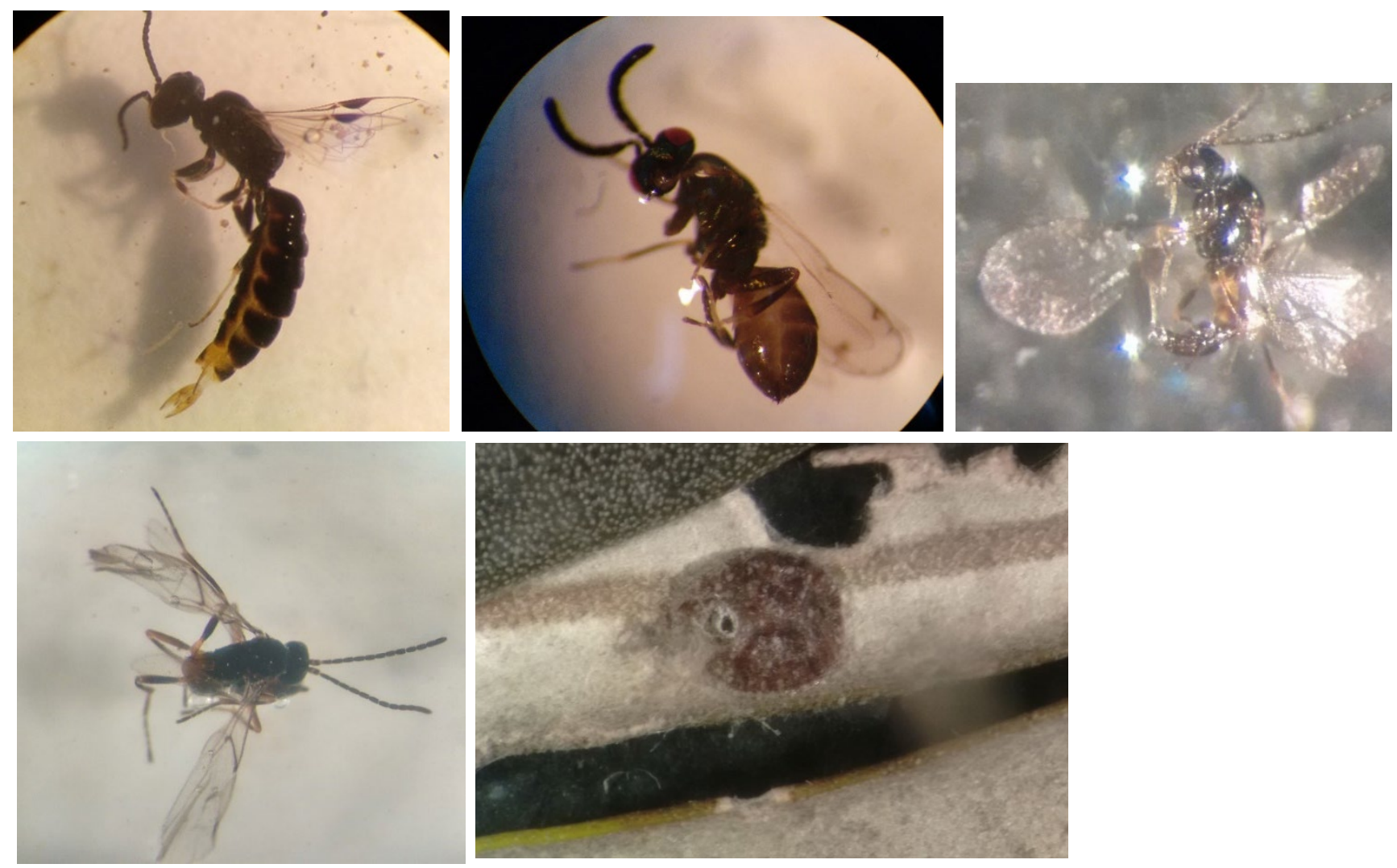

Figura 5. Himenópteros parasitoides catalogados en el campus. De izquierda a derecha: Aphidius sp, Torymus notatus, Trioxys curvicaudus, Diaeretiella rapae, Saissetia oleae con el orificio de salida del parasitoide Methaphycus helvolus. 
Con la información aportada, aún siendo datos preliminares, podemos concluir que la vegetación ornamental del campus, mantiene una importante variedad de entomofauna asociada entre cuyas especies se establece una red de interacciones tróficas que puede ser mayor de la aquí presentada ya que este es el resultado de 6 meses de muestreo. Con los datos aportados se pretende facilitar el primer registro de especies fitófagas que, en la actualidad, y de forma variable según los años, causan daño de diversa consideración en las plantas del campus y también de la de sus enemigos naturales que, de un modo inadvertido, pero eficaz están controlando de manera natural el crecimiento de alguna de las poblaciones plaga. Estos datos deben servir de guía para que se mantengan y establezcan nuevas medidas que frenen la pérdida de las especies de polinizadores, depredadores y parasitoides de especies que pueden ser potencialmente plagas de esta zona ajardinada.

\section{AGRADECIMIENTOS}

Agradecer la ayuda en la identificación de parte del material a los siguientes entomólogos especialistas: Dr. J. L. Nieves Aldrey (himenópteros gallícolas), Dr. S. Bordera (Hymenoptera, Ichneumonidae), Dr. J. R. Verdú (Coleoptera), Dra. C. Ornosa (Hymenoptera Apoidea) y Dr. Pedro del Estal (Hemiptera). Agradecer también a J.L. Romeu, la información facilitada acerca de los tratamientos aplicados y del mantenimiento de la zona ajardiana del campus.

\section{REFERENCIAS}

Aguiar, A. (2013) New records of aphids (Hemiptera: Aphidoidea) from Madeira and Azores Archipelagos. Entomologist's monthly magazine, 149: 235-254

Barrientos. J.A. (2004), Curso práctico de Entomología. Univ. Autónoma de Barcelona, CIBIO Universidad de Alicante, Asociación española Entomología. España. $947 \mathrm{pp}$.
García, A., Barniol, J., Argemí, C., Argimon, X., Caballero, S., Camp F., Camps, J., Fernández, J.M., Garcia, S., Garcia, S., Giné, J., Lacambra, C., Maluenda, J., Navarro, P., Nerhing, R., Plaza, T., Poch, J.M., Rojo. M., Solanes, J. Sorribas, R., Tena, J., Vives de Quadras, J.M. (2015) Llibre blanc sobre control de plagues en espais verds. Generalitat de Catalunya, Departament d'Agricultura, Ramaderia, Pesca i Alimentació, Servei de Sanitat Vegetal. España. 154pp

Bella, S., Mifsud, D., Perez Hidalgo, N., \& Barbagallo, S. (2009). Greenidea ficicola: Is It an Example of Rapid Colonization Due To Climatic Changes?. Redia, 92:129-139.

Bellmann, H. (2003). Guía de campo Blume insectos. Blume. España. 191 pp.

Ben-Dov, Y. (2011). The scale insects (Hemiptera: Coccoidea) of Israel checklist, host plants, zoogeographical considerations and annotations on species. Israel Journal of Entomology, 41-42:21-48.

Bermejo, J (2011). Base de datos con imágenes y fichas actualizadas de las plagas agrícolas de España. Agrologica Servicios Agricola, S.L. España. Disponible en: http:// www.agrologica.es/[Consultado 17-3-2018]

Bignon J-J. (2006). Observar los insectos. Tikal. España 223 pp.

Biradar, A.P. \& Shaila, H.M. (2004). Management of aphid, Aphis punicae Passerini (Homoptera; Aphididae) in pomegranate. Pest Management In Horticultural Ecosystems, 10(2):157-159.

Blackman, R.L. \& Eastop, V.F. (2000). Aphids on the World's Crops. An Identification and Information Guide. JohnWiley \& Sons. Chichester. 414 pp.

CABI (2019). Invasive Species Compendium. Wallingford, UK: CAB International. www.cabi.org/isc.

Caleca, V. (2010). First record in Algeria of two eulophid wasps: Closterocerus chamaeleon (Girault) and its host, the eucalyptus gall wasp Ophelimus maskelli (Asmead) (Hymenoptera Eulophidae). Naturalista siciliano, 34(12):201-206.

Calvo, J., Bolckmans, K., Stansly, P. A., \& Urbaneja, A. (2008). Predation by Nesidiocoris tenuis on Bemisia tabaci and injury to tomato. BioControl, 54(2):237246. 
Chávez-Moreno, C.K., Tecante, A, Casas, A, \& Claps, L.E. (2011). Distribution and Habitat in Mexico of Dactylopius Costa (Hemiptera: Dactylopiidae) and their Cacti Hosts (Cactaceae: Opuntioideae). Neotropical Entomology, 40(1):62-71.

Chueca, P. \& Moltó, E. (2012). "Plagas y Enfermedades”. En "Gestión Integrada de Plagas de Cítricos”, Ed. por Urbaneja A., Catalán J., Tena A., Jacas, J., http:// gipcitricos.ivia.es, 2012.

Climent, J. M. L. (1990a) Homoptera I. Cochinillas de los cítricos y su control biológico. Pisa. Valencia. 260 pp.

Climent, J.M.L. (1990b). Homoptera II. Pulgones de los cítricos y su control biológico. Pisa. Valencia 170 pp

Climent, J.M.L. (1992). Homoptera III. Moscas blancas y su control biológico. Pisa.Valencia. 203 pp

Cole, R. A. (1997). Comparison of feeding behaviour of two Brassica pests Brevicoryne brassicae and Myzus persicae on wild and cultivated brassica species, Entomologia Experimentalis et Applicata, 85(2):135143.

De Liñan, V. C., Baragaño Galán, .J, Notario Gómez, A., Beitia Crespo, F., Bielza Lino, P., Castesana Estrada, L., Contreras Gallego, J., Del Estal Padillo, P., Esteban Durán, J., Garrido Vivas, A., Jiménez Álvarez, A., Lacasa Plasensia, A., Sánchez Sánchez, J.A. Yela García, J.L., Vińuela Sandoval, E. \& Jacas Miret, J. Entomología Agroforestal Insectos y que dañan montes, cultivos y jardines. Ediciones Agrotécnicas S.L. España. 1309 pp

Debach, P. (1959). New Species and strains of Aphytis (Hymenoptera, Eulophidae) Parasitic on the California Red Scale, Aonidiella Aurantii (Mask.), in the Oriental. Annals of the Entomological Society of America, 52(4):354-362.

Denmark, H.A., Fasulo, R. \& Funderburk, J.E. (2004). Cuban laurel thrips, Gynaikothrips ficorum (Marchal) (Insecta: Thysanoptera: Phlaeothripidae). University of Florida, U.S.A.

Dhahri, S., Ben Jamaa, M.L. \& Lo Verde, G. (2010) First Record of Leptocybe invasa and Ophelimus maskelli Eucalyptus Gall Wasps in Tunisia. Tunisian journal of plant protection, 5(5(2)):231-236.

Diaz, B. M. \& Fereres, A. (2005) Life Table and Population Parameters of Nasonovia ribisnigri (Homoptera: Aphididae) at Different Constant Temperatures, Environmental Entomology, 34(3):527534.
Dixon, A. F. G. (1958). The escape responses shown by certain aphids to the presence of the coccinellid Adalia decempunctata (L.). Transactions of the Royal Entomological Society of London, 110:319-334.

Douglas, N. \& Krüger, K. (2008). Transmission efficiency of Grapevine leafroll-associated virus 3 (GLRaV-3) by the mealybugs Planococcus ficus and Pseudococcus longispinus (Hemiptera: Pseudococcidae). European Journal of Plant Pathology, 122(2):207-212.

Egerer, M. H., Liere, H., Lin, B. B., Jha, S., Bichier, P., \& Philpott, S. M. (2018). Herbivore regulation in urban agroecosystems: Direct and indirect effects. Basic and Applied Ecology, 29:44-54.

Fisher, D. (2007). Apple weevil (Otiorhynchus cribricollis) pest of viticulture Common names. Departament of Agriculture and Food. Australia.

García-Marí, F., Llorens Climent, J.L., \& Costa Comelles, J.F.F. (1991). Ácaros de las plantas cultivadas y su control biológico, Pisa. 188pp.

Hall, R.W. \& Townsend, A.M. (1987) Suitability of Ulmus wilsoniana, the 'Urban' Elm, and Their Hybrids for the Elm Leaf Beetle, Xanthogaleruca luteola (Müller) (Coleoptera: Chrysomelidae). Environmental Entomology, 16(5):1042-1044.

Hall, R.W. \& Young, C.E., (1986) Host Suitability of Three Asiatic Elms to the Elm Leaf Beetle (Xanthogaleruca luteola) (Coleoptera: Chrysomelidae). Journal of Environmental Horticulture, 4(2):44-46.

Heppner, J. B. (1993). Citrus Leafminer. The Institute of Food and Agricultural Sciences. U.S.A

Johnson, M. W., \& Hara, A. H. (1987) Influence of Host Crop on Parasitoids (Hymenoptera) of Liriomyza spp. (Diptera: Agromyzidae). Environmental Entomology, 16(2):339-344

Kamel, N. (2012) Aphid fauna (Hemiptera Aphididae) and their host association of Chott Mariem, coastal area of Tunisia. Annuals of Biological Research, 3(1):111.

Kang, L., Chen, B., Wei, J.N. \& Liu, T.X. (2009) Roles of Thermal Adaptation and Chemical Ecology in Liriomyza Distribution and Control. Annual Review of Entomology, 54(1):127-145.

Khasawinah, A. M. A., \& Talhouk, A. S. (2009). The Fig Wax Scale, Ceroplastes rusci (Linn.). Zeitschrift Für Angewandte Entomologie, 53(1-4):113-131. 
Leraut, P. (2007) Insectos de España y Europa. Lynx. España. 523pp

Melia, A., Cabezuelo, P. \& Fernández De córdoba, J. (1993). Incidencia de pulgones (Homoptera, Aphididae) en encinares de Córdoba. Boletín Sanidad Vegetal y Plagas, 19: 355-360.

Moret, A. \& Martí, N. (2006). Guía de insectos perjudiciales y beneficiosos para la agricultura. Omega. España. 279 pp

Muñoz López, C., Pérez Fortea, V., Cobos Suárez, P., Hernández Alonso, R. \& Sánchez Peña, G. (2011). Sanidad Forestal. Guia en imágenes de plagas, enfermedades y otros agentes presentes en los bosques. Mundi-Prensa. España. 575pp

Myers, P., R. Espinosa, C. S. Parr, T. Jones, G. S. Hammond, \& T. A. Dewey ( 2018) The Animal Diversity Web (online). Accessed at https:// animaldiversity.org.

Nardi, F., Carapelli, A., Dallai, R., Roderick, G. K., \& Frati, F. (2005). Population structure and colonization history of the olive fly, Bactrocera oleae (Diptera, Tephritidae). Molecular Ecology 14(9):2729-2738.

Parr, C.S., Wilson, N., Leary, P., Schulz, K.S., Lans, K., Walley, L., Hammock. J.A., Goddard, A., Rice, J., Studer, M., Holmes, J.T.G. \& Corrigan, R.J. (2014). The Encyclopedia of Life v2: Providing Global Access to Knowledge About Life on Earth.

Pellizzari, G., Porcelli, F., Convertini., S. \& Marotta, S. (2012). Description of nymphal instars and adult female of Kermes vermilio Planchon (Hemiptera, Coccoidea, Kermesidae), with a synopsis of the European and Mediterranean species. Zootaxa, 3336:36-50.

Pérez Hidalgo, N. (2015). La tribu Fordini (Hem.: Aphididae: Eriosomatinae) en la península Ibérica: un enfoque multidisciplinar. Tesis Doctoral, España, 140 pp.

Pérez Hidalgo, N., Sousa-Silva, P \& Estal, P. (2009). La presencia del pulgón asiático Greenidea ficicola (Takahashi) [Aphididae: Greenideinae: Greenideini] en España. Boletin de Sanidad Vegetal Plagas. 35:581-584.
Pike, K. S., Starý, P., Miller, T., Allison, D., Graf, G., Boydston, L., \& Gillespie, R. (1999). Host range and habitats of the aphid parasitoid Diaeretiella rapae (Hymenoptera: Aphidiidae) in Washington State. Environmental Entomology, 28(1):61-71.

Rodrigo, E. \& García-Marí, F. (1992) Ciclo biológico de los diaspinos de cítricos Aonidiella aurantii (Mask.), Lepidosaphes beckii (Newm.) y Parlatoria pergandei (Comst.) en 1990. Boletín Sanidad Vegetal de Plagas, 18:31-44.

Rodrigo Santamalia, ME., Laborda Cenjor, R., SanchezDomingo, A., González-Rey, S., Bertomeu, S., Xamani Monserrat, P. \& Hernández-Suárez, E. (2017). Primer registro de Josephiella microcarpae Beardsley \& Rasplus, 2001 (Hymenoptera, Agaonidae) en Ficus microcarpa L. en España peninsular. Boletín de la Sociedad Entomológica Aragonesa. 60:390-392.

Rojo, S., Gilbert, M.A., Marcos-García, M.A., Nieto, J.M. \& Durante, P. (2003). A world review of predatoru hoverflies (Diptera: Syrphidae: syrphinae) and their prey. CIBIO. España. 219 pp.

Rotheray, G.E.; Gilbert, F.S. (2011). The Natural History of Hoverflies. Forrest Text. Londres. 333pp.

Sanchez, J. A. (2008). Zoophytophagy in the plantbug Nesidiocoris tenuis. Agricultural and Forest Entomology, 10(2):75-80.

Senior, L. J., McEwen, P. K., McEwen, P., New, T., \& Whittington, A. (2001). The use of lacewings in biological control. En: McEwen, P., New, T., \& Whittington, A. (eds), Lacewings in the crop environment. Cambridge University Press, Cambridge. Pp 296-302.

Stegmaier, C.E. (1966) Host Plants and Parasites of Liriomyza trifolii in Florida (Diptera: Agromyzidae). The Florida Entomologist, 49(2):75-89.

Stenberg, J. (2017). A conceptual framework for integrated pest management. Trends in Plant Science, 22:759-769.

Thomas, M.C., Heppner, J.B., Woodruff, R.E., Weems, H. V, Steck, G.J., Fasulo, T.R. \& Steck, F. (2013). Mediterranean Fruit Fly, Ceratitis capitata (Wiedemann) (Insecta: Diptera: Tephritidae). DPI Entomology Circulars, 4:230-273. 
Urbaneja, A., Montón, H., \& Mollá, O. (2009). Suitability of the tomato borer Tuta absoluta as prey for Macrolophus pygmaeus and Nesidiocoris tenuis. Journal of Applied Entomology, 133(4):292-296.

Valente, C., \& Hodkinson, I. (2009) First record of the red gum lerp psyllid, Glycaspsi brimblecombei, in Europe. Journal of Applied Entomology, 133:315-317.

Velasco, L.R.I., \& Walter, G.H. (1992). Availability of Different Host Plant Species and Changing Abundance of the Polyphagous Bug Nezara viridula (Hemiptera: Pentatomidae). Environmental Entomology, 21(4):751759.
Wysoki, M. (1987). A bibliography of the pyriform scale, Protopulvinaria pyriformis (Cockerell) (homoptera: coccidae), up to 1986. Phytoparasitica, 15(1):73-77.

Zehnder, G.W. \& Trumble, J.T. (1984). Host Selection of Liriomyza Species (Diptera: Agromyzidae) and Associated Parasites in Adjacent Plantings of Tomato and Celery. Environmental Entomology 13(2):92. 
Este número ha recibido una ayuda del Vicerrectorado de Investigación y Transferencia de Conocimiento de la Universidad de Alicante
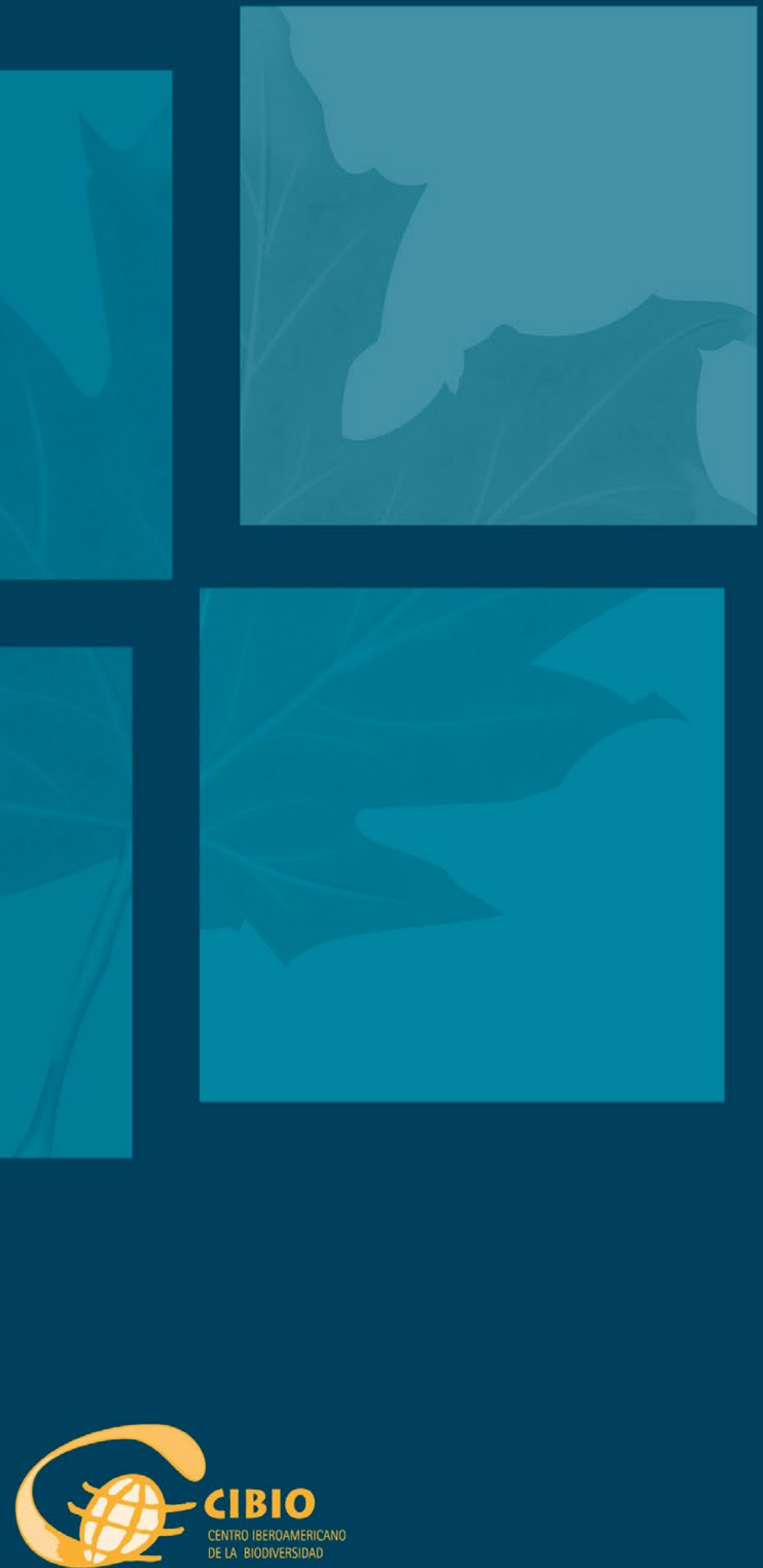